Check for updates

Cite this: RSC Adv., 2017, 7, 20833

Received 6th November 2016

Accepted 3rd April 2017

DOI: 10.1039/c6ra26375e

rsc.li/rsc-advances

\section{Edge effect detection for real-time cellular analyzer using statistical analysis}

\begin{abstract}
Yinghao Chen, ${ }^{a}$ Shan Chen, ${ }^{a}$ Tianhong Pan (D) *a and Xiaobo Zou (D) ${ }^{b}$
Real time cellular analyzers (RTCA) are widely used to test the cytotoxicity of chemicals. However, there are some uncontrollable factors, which are detrimental to the experimental quality. One of the fundamental issues is the edge effect. Abnormal time-dependent cellular response curves (TCRCS) are observed when the wells are located at the edge of the E-plate. In this paper, the Smirnov test was used to detect the edge effect. The average normalized cell index $(\mathrm{NCl})$ of the negative control located in the inner wells was taken as the standard. Thereafter, all TCRCs were divided into several intervals, and their corresponding empirical distribution functions (EDFs) of the mean $\mathrm{NCl}$ and $\mathrm{NCl}$ located at the edge wells were calculated, and hypothesis testing was used to determine the differences of the EDFs. The experimental results evaluated the performance of the proposed algorithm. This framework provided a systematic method for edge-effect detection.
\end{abstract}

\section{Introduction}

To assess the hazards of industrial chemicals, consumer products, pharmaceuticals, and environmental contaminants, animal tests are generally adopted by the Globally Harmonized System (GHS) classification and human health risk assessments. ${ }^{1,2}$ However, the cost of animal experiments is high and the understanding of the mechanism of action remains limited. Cell based in vitro assay is an alternative to animal testing in safety/hazard assessments. ${ }^{3-6} \mathrm{~A}$ popular instrument in cell-based in vitro assay is the xCELLigence real-time cell analyzer (RTCA), which allows for uninterrupted, noninvasive, label-free, and real-time analysis of cells over the course of an experiment. ${ }^{7}$ It has been widely used in many different research fields, such as drug discovery, water quality monitoring, and toxicology investigations. ${ }^{8-12}$ However, although researchers use RTCA as a tool, the experimental quality is not always noted.

In the RTCA experiment, the cellular response in the edge well of the E-plate is usually different from that of the inner well, especially in the E-plate $\times 96$ and $\times 384$ formats, which is described as the edge effect. There are two main reasons why the edge effect appears in RTCA experiments: one reason is that the evaporation of long-term incubation causes the edge effect (evaporation effect), because the evaporation efficiency of water in the edge well is higher than that in the inner well; and the other reason is that the temperature of the edge well reaches the desired incubation temperature faster than that of the inner well owing to plate stacking (temperature effect). ${ }^{13}$ To weaken the

${ }^{a}$ School of Electrical and Information Engineering, Jiangsu University, Zhenjiang, Jiangsu, 212013, China. E-mail: thpan@live.com; Tel: +86-15805298357b

${ }^{b}$ School of Food and Biological Engineering, Jiangsu University, Zhenjiang, Jiangsu 212013, China edge effect, Lundholt and Scudder ${ }^{14}$ pre-incubated newly seeded plates in ambient conditions (air at room temperature), which resulted in an even distribution of the cells in each well. However, the result of this method is not stable and would be invalid for long-term cultivation $(>24 \mathrm{~h})$. Furthermore, a special anti-volatile lid has been developed to restrain the edge effect, ${ }^{15}$ but the performance is limited. Malo and Hanley ${ }^{\mathbf{1 6}}$ proposed a statistical method to optimize the configuration of the reference wells for reducing the positional effects of wells within plates. In a nutshell, those methods are designed to reduce the edge effect on the experimental process or device, nevertheless, they cannot completely eliminate the edge effect. Furthermore, there is no effective method to identify the edge effect.

To avoid the edge effect, researchers tend to abandon the data located at edge wells, which decreases the testing efficiency of the RTCA and will make a lot of waste, especially in E-plate $\times 96$ formats $(37.5 \%)$. Furthermore, researchers manually screen the edge effect, which is time consuming and can result in inaccuracies. In this work, the Smirnov test was used to determine the edge effect. Compared with manual selection, the proposed method is efficient and reliable.

This paper has been organized as follows: in Section 2, the problem of edge effect has been described. In Section 3, the Smirnov test was applied to identify the edge effect with related variables. In Section 4, several RTCA experiments were used to validate the proposed method. Finally, Section 5 included concluding remarks.

\section{Problem statement}

In this study, the xCELLigence RTCA system with E-plate $\times 384$ formats was selected as the experimental equipment and the 


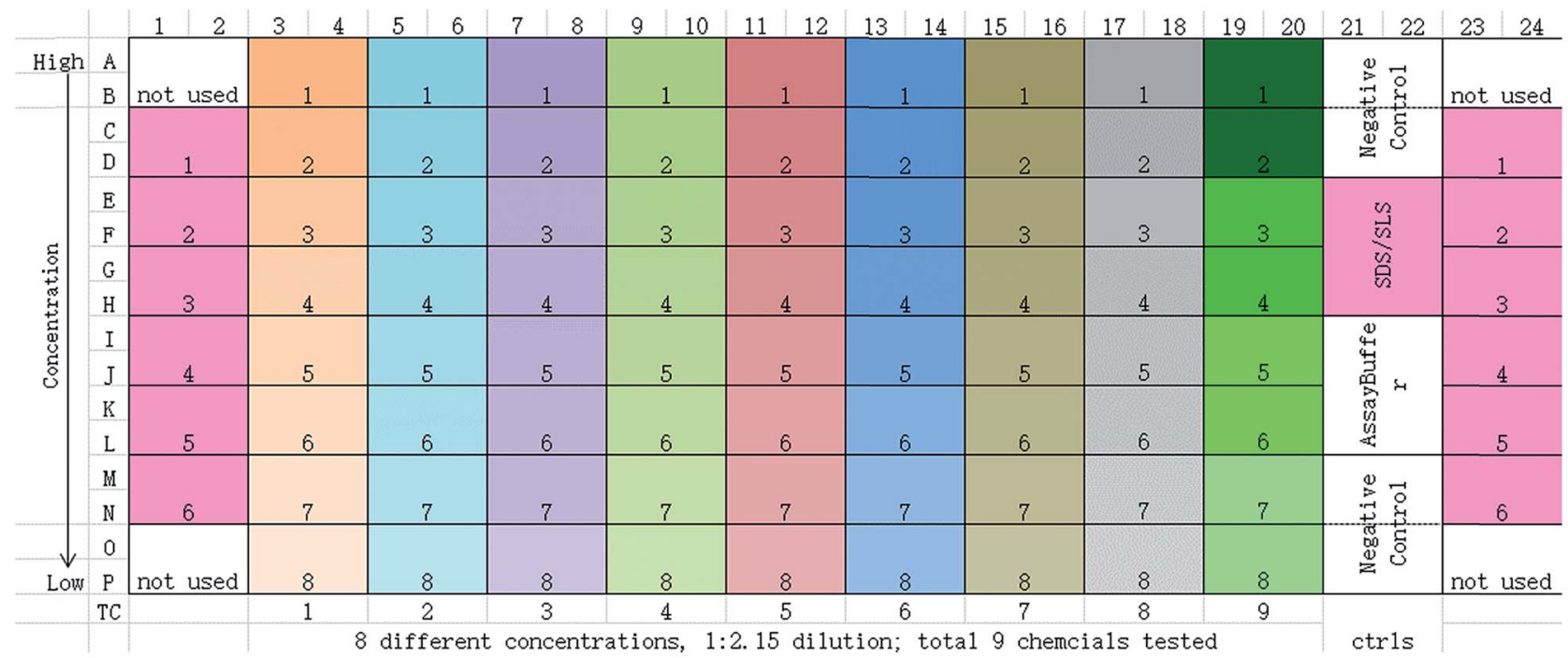

(a)

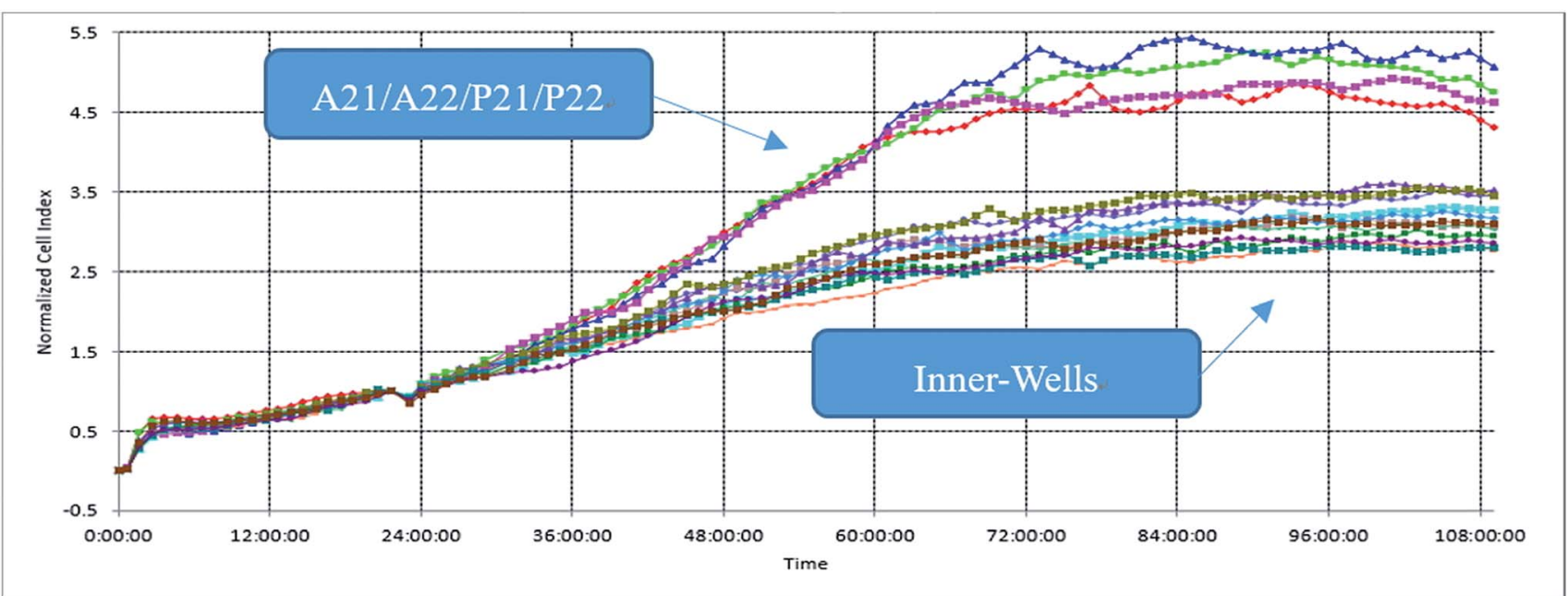

(b)

Fig. 1 Experiment and its edge effect. (a) The schematic diagram of RTCA. (b) TCRCs of negative control.

schematic diagram is shown in Fig. 1(a). ${ }^{17}$ The basic principle of the RTCA is to monitor the cell population changes in electrode impedance induced by the interactions between testing cell numbers and electrodes.

The procedure of the cytotoxicity assay is as follows: first, a certain cell line was added to each well of the $\times 384$ E-plate and incubated in the culture medium about $24 \mathrm{~h}$; second, different chemicals with different concentrations were added to the E-plate, which followed a particular protocol: the same chemical was added to every two columns of wells, and the same concentration of chemical decreasing from top to bottom was added to every two rows of wells. According to this protocol, each concentration of each chemical was repeated four times (for example, as shown in Fig. 1(a), A3, A4, B3, and B4 wells have the same culture environment; C3, C4, D3, and D4 have the same culture environment) to enhance the robustness of the experiment. An experiment lasted about $96 \mathrm{~h}$. The timedependent cellular response profiles were dynamically

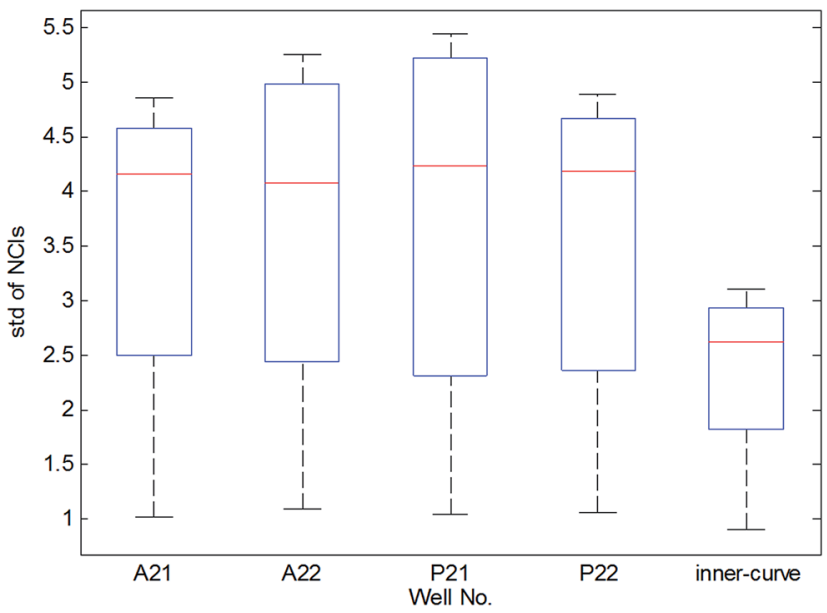

Fig. 2 Box plots of standard deviation (STD) of NCls located at edge and inner wells. 
Table 1 Edge effect detection based on the Smirnov test

\begin{tabular}{|c|c|c|}
\hline 1 & Initialize & $\begin{array}{l}D_{n, 1-\alpha}, M=12, T=72, j=0, m=1, \delta_{0}=0, k=1, \\
F_{0}=0\end{array}$ \\
\hline 2 & Compute & $\overline{\mathrm{NCI}}(t)=\frac{1}{M} \sum_{i=1}^{M} \mathrm{NCI}^{i}(t), \quad t=1,2, \ldots, \quad T$ \\
\hline 3 & Repeat & \\
\hline 4 & Repeat & $\begin{array}{l}\text { For } t=1 ; t \leq T ; t++, \text { if } \mathrm{NCI}^{m}(t) \in\left[\delta_{j}, \delta_{j}+1\right), \nu_{j}^{m}= \\
\nu_{j}^{m}+1 \text {, end if, if } \overline{\operatorname{NCI}}(t) \in\left[\delta_{j}, \delta_{j}+1\right), \nu_{j}=\nu_{j}+1, \\
\text { end if, end for }\end{array}$ \\
\hline 5 & & $\delta_{j+1}=\delta_{j}+1$ \\
\hline 6 & & $j=j+1$ \\
\hline 7 & Until & $j=\operatorname{fix}\left(\max \left(\left\{\overline{\mathrm{NCI}}(t), \mathrm{NCI}^{m}(t)\right\}_{t=t_{0}}^{T}\right)\right)$ \\
\hline 8 & Compute & $F_{k}^{m}=\frac{1}{T} \sum^{k} \nu_{j}^{m}$ \\
\hline & & $\left\{\begin{array}{l}j=0 \\
1\end{array}\right.$ \\
\hline & & $\begin{array}{l}\bar{F}_{k}=\frac{1}{T} \sum_{j=0} \bar{\nu}_{j} \\
\max \left|\bar{F}_{k}-F_{k}^{m}\right|\end{array}$ \\
\hline 9 & & $\begin{array}{l}\text { If } D^{m} \geq D_{n, 1-\alpha} \text {, these is an edge effect and } \\
\text { discard the } m^{\text {th }} \text { TCRC, else keep the } m^{\text {th }} \text { TCRC }\end{array}$ \\
\hline 10 & & $m=m+1$ \\
\hline 11 & Until & $m=4$ \\
\hline 12 & End & \\
\hline
\end{tabular}

recorded in RTCA, which was termed the Normalized Cell Index (NCI) (shown in Fig. 1(b)). In the cell based in vitro assay, the data after the cellular exposure was important (from 24 to $96 \mathrm{~h}$ ), so the total sampling time was $72 \mathrm{~h}$.

As shown in Fig. 1(b), the time-dependent cellular response curves (TCRCs) of A21/A22/P21/P22 (edge wells) were significantly different from the TCRCs of inner wells. NCIs of the inner wells were lesser than those of edge wells, this was named the edge effect. To describe the differences, the standard deviations of NCIs in the sampling interval located at edge/inner well were calculated and shown in Fig. 2. The mean NCIs of the inner wells were set as standard. The boxplots of edge wells were obviously different from those of the inner wells. As mentioned previously, the abnormal TCRCs located at the edge wells were screened manually. The automatic detection of the edge effect was the key issue in the RTCA experiment. To develop a validated method, the data of negative control were selected as examples, since cells without chemicals grow naturally in the culture medium. This eliminates the error cause by different cell growth environment.

\section{Smirnov test method}

To eliminate the effect of cell seeding populations, the cell index (CI) as normalized as follows:
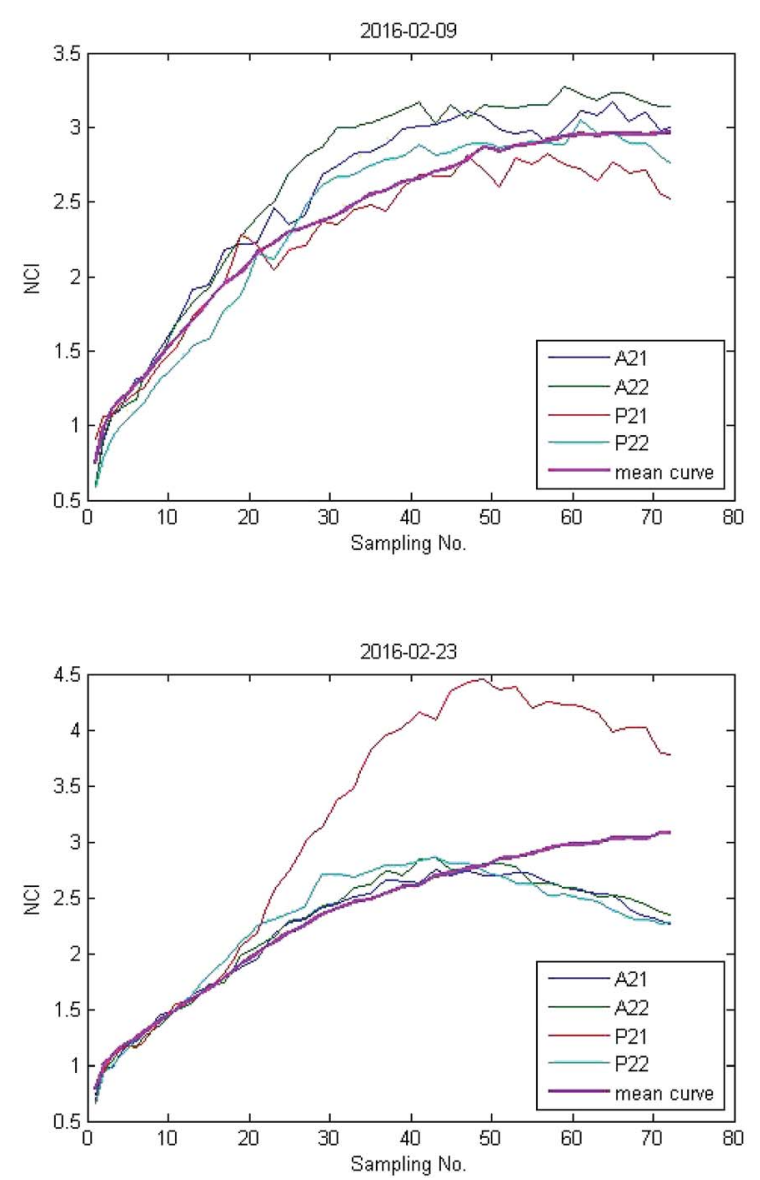

Fig. $3 \mathrm{NCls}$ of four experiments. 

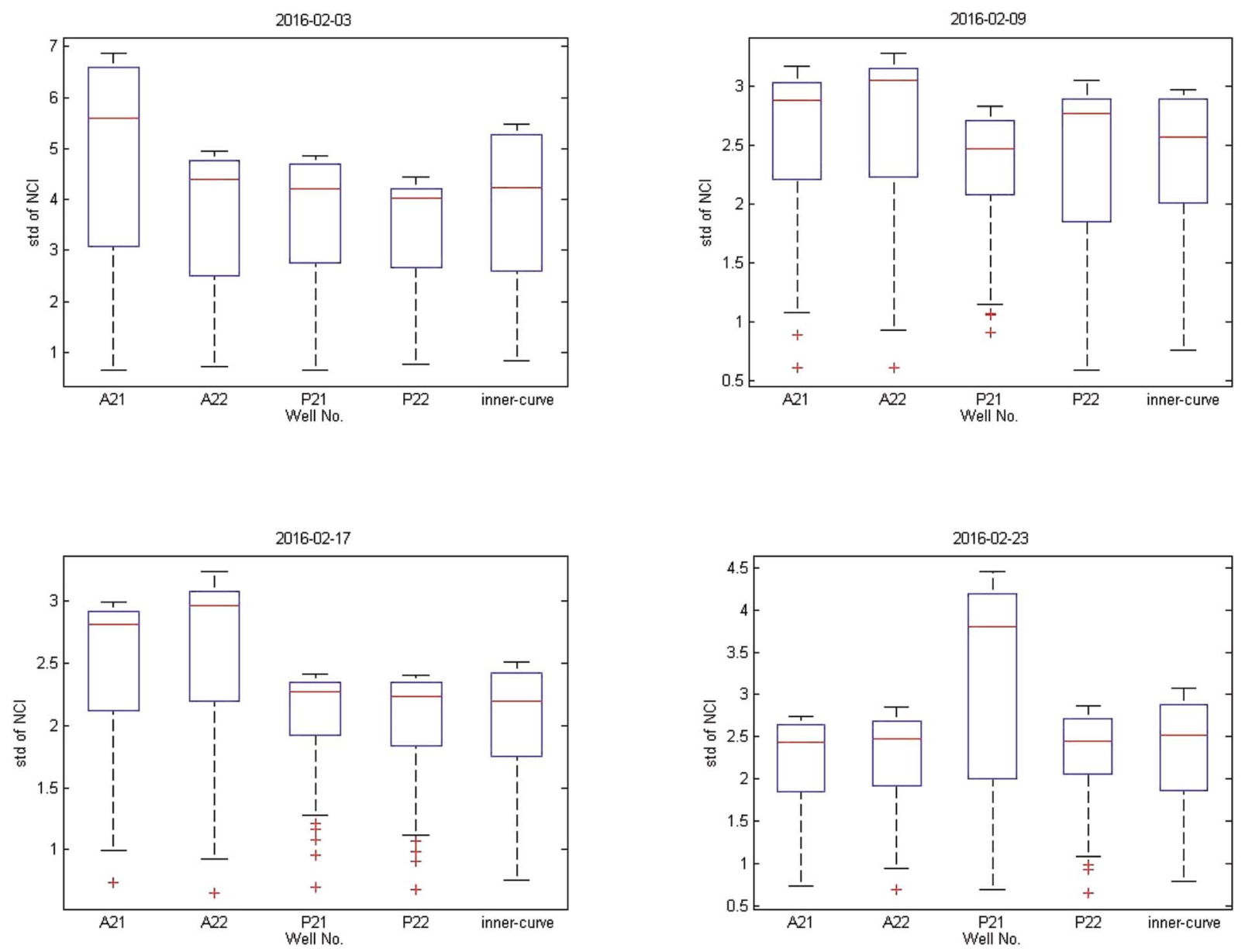

Fig. 4 Box plots of four experiments.

Table 2 The empirical distribution function of experiment 1

\begin{tabular}{|c|c|c|c|c|c|c|c|c|c|c|}
\hline Range interval & \multicolumn{5}{|c|}{ Cumulative frequency } & \multicolumn{5}{|c|}{ Empirical distribution function } \\
\hline$[1,2)$ & 14 & 13 & 15 & 15 & 20 & 0.014 & 0.014 & 0.014 & 0.014 & 0.014 \\
\hline$[2,3)$ & 10 & 11 & 10 & 10 & 37 & 0.208 & 0.194 & 0.222 & 0.222 & 0.292 \\
\hline$[3,4)$ & 10 & 11 & 10 & 10 & 14 & 0.347 & 0.347 & 0.361 & 0.361 & 0.806 \\
\hline
\end{tabular}

$$
\mathrm{NCI}(t)=\frac{\mathrm{CI}(t)}{\mathrm{CI}(0)}, \quad t=1,2, \ldots, \quad T
$$

where NCI (normalized cell index) is the ratio of CI at a particular point of time to the CI at the time of exposure. ${ }^{18} t$ is the sampling sequence number, $T$ is the quantity of sampling points and is set as 72 because the sampling interval is $1 \mathrm{~h}$ for 3 d. The normalization procedure was embedded in the RTCA system and NCI value could be directly exported.

As shown in Fig. 1(b), the TCRCs located at inner wells were similar to each other, and different from the TCRCs located at edge wells (denoted asNCI ${ }^{m}, m=1,2,3,4$ ). Here, NCIs of inner wells were averaged as a standard:
Table 3 Results of experiment 1 with $\alpha=0.05$

\begin{tabular}{lllll}
\hline Edge-well & A21 & A22 & P21 & P22 \\
\hline$D^{m}=\max \left|\bar{F}_{k}-F_{k}{ }^{m}\right|$ & 0.514 & 0.500 & 0.500 & 0.500 \\
$D^{m}>D_{36,1-0.05}$ & Yes & Yes & Yes & Yes \\
Edge effect & Yes & Yes & Yes & Yes \\
\hline
\end{tabular}

$$
\overline{\operatorname{NCI}}(t)=\frac{1}{M} \sum_{i=1}^{M} \operatorname{NCI}^{i}(t), \quad t=1,2, \ldots, \quad T
$$

where $i=1,2, \ldots, M$ is the number of TCRCs located at inner wells (shown in Fig. 1, $M=12$ ). 
It is difficult to distinguish the difference between $\overline{\mathrm{NCI}}$ and $\mathrm{NCI}^{m}$ directly, because TCRC is one type of time series. To develop a validated method, the empirical distribution of NCI was used and the range interval was set as:

$$
\left\{\left[\delta_{j}, \delta_{j}+1\right)\right\}_{j=0}{ }^{J}
$$

where $\delta_{0}=0, j=0,1, \ldots, J$ is the number of range interval and

$$
J=\operatorname{fix}\left(\max \left(\left\{\overline{\mathrm{NCI}}(t), \mathrm{NCI}^{m}(t)\right\}_{t=1}^{T}\right)\right)
$$

where $\operatorname{fix}(\cdot)$ is rounding math function.

For each TCRC, the cumulative frequency of each interval was calculated as follows:

$$
\nu_{j}=\left\{\begin{array}{l}
\nu_{j}+1 \quad \mathrm{NCI}(t) \in\left[\delta_{j}, \delta_{j}+1\right) \\
\nu_{j} \text { otherwise }
\end{array} \quad j=0,1, \cdots, J\right.
$$

Through the interval segmentation and frequency accumulation, the time series NCI was transformed into independent random variables. Then the corresponding empirical distribution function (EDF) of each TCRC was computed:

$$
\left\{\begin{array}{l}
F_{0}=0, \\
F_{k}=\frac{1}{T} \sum_{j=0}^{k} \nu_{j}, \quad k=1,2,3, \ldots, J
\end{array}\right.
$$

As a result, the EDFs of NCI of each edge-well $\left(F^{m}\right)$ and mean NCI of inner wells $(\bar{F})$ were obtained. The Smirnov test was used to determine whether the TCRC's EDFs of the edge wells were equal to the mean TCRC's EDFs, i.e.:

$$
\begin{aligned}
& H_{0}: F^{m}=\bar{F} \\
& H_{1}: F^{m} \neq \bar{F}
\end{aligned}
$$

where, $H_{0}$ is the null hypothesis and $H_{1}$ is the alternative hypothesis, $\bar{F}$ and $F^{m}(m=1,2,3,4)$ are the EDFs of the mean TCRC and the four edge TCRCs, respectively.

Table 4 Results of four experiments with $\alpha=0.05$

\begin{tabular}{llll}
\hline Experiment & Edge-well & $D^{m}$ & Edge-effect \\
\hline \multirow{2}{*}{$2016-02-03$} & A21 & 0.514 & Yes \\
& A22 & 0.500 & Yes \\
& P21 & 0.500 & Yes \\
$2016-02-09$ & P22 & 0.500 & Yes \\
& A21 & 0.306 & Yes \\
& A22 & 0.542 & Yes \\
$2016-02-17$ & P21 & 0.292 & Yes \\
& P22 & 0.202 & No \\
& A21 & 0.486 & Yes \\
& A22 & 0.516 & Yes \\
$2016-02-23$ & P21 & 0.186 & No \\
& P22 & 0.186 & No \\
& A21 & 0.284 & Yes \\
& A22 & 0.486 & Yes \\
& P21 & 0.284 & Yes \\
& P22 & 0.284 & Yes
\end{tabular}

To compare the EDFs of two different samples without assuming any underlying parametric model for the sample, i.e. it is a nonparametric test, ${ }^{19}$ the Smirnov test is taken for consideration in this work.

The statistical magnitude of Smirnov test $D^{m}$ was given by:

$$
D^{m}=\max \left|\bar{F}_{k}-F_{k}{ }^{m}\right|
$$

$D^{m}$ is compared with the critical region $D_{n, 1-\alpha}$ in the Kolmogorov distribution quantile table. If the value of $D^{m}$ is out of the critical region of $D_{n, 1-\alpha}$, hypothesis $H_{0}$ is rejected and the alternate hypothesis $H_{1}$ is accepted. In the Kolmogorov distribution quantile table, $n$ is defined as:

Table 5 Results of four experiments with $\alpha=0.1$

\begin{tabular}{llll}
\hline Experiment & Edge-well & $D^{m}$ & Edge-effect \\
\hline $2016-02-03$ & A21 & 0.514 & Yes \\
& A22 & 0.500 & Yes \\
& P21 & 0.500 & Yes \\
$2016-02-09$ & P22 & 0.500 & Yes \\
& A21 & 0.306 & Yes \\
& A22 & 0.542 & Yes \\
& P21 & 0.292 & Yes \\
& P22 & 0.202 & Yes \\
& A21 & 0.486 & Yes \\
& A22 & 0.516 & Yes \\
& P21 & 0.186 & No \\
& P22 & 0.186 & No \\
& A21 & 0.284 & Yes \\
& A22 & 0.486 & Yes \\
& P21 & 0.284 & Yes \\
& P22 & 0.284 & Yes
\end{tabular}

Table 6 Results of the actual experiments

\begin{tabular}{llllll}
\hline Edge-well & $D^{m}$ & Edge-effect & Edge-well & $D^{m}$ & Edge-effect \\
\hline A1 & & & & & \\
A2 & 0.514 & Yes & P1 & 0.514 & Yes \\
A3 & 0.202 & No & P2 & 0.202 & No \\
A4 & 0.186 & No & P3 & 0.306 & Yes \\
A5 & 0.186 & No & P4 & 0.486 & Yes \\
A6 & 0.306 & Yes & P5 & 0.368 & Yes \\
A7 & 0.542 & Yes & P6 & 0.368 & Yes \\
A8 & 0.202 & No & P7 & 0.486 & Yes \\
A9 & 0.202 & No & P8 & 0.202 & No \\
A10 & 0.186 & No & P9 & 0.386 & Yes \\
A11 & 0.186 & No & P10 & 0.128 & No \\
A12 & 0.070 & No & P11 & 0.128 & No \\
A13 & 0.070 & No & P12 & 0.292 & Yes \\
A14 & 0.284 & Yes & P13 & 0.292 & Yes \\
A15 & 0.486 & Yes & P14 & 0.306 & Yes \\
A16 & 0.128 & No & P15 & 0.128 & No \\
A17 & 0.284 & Yes & P16 & 0.128 & No \\
A18 & 0.306 & Yes & P17 & 0.070 & No \\
A19 & 0.306 & Yes & P18 & 0.070 & No \\
A20 & 0.128 & No & P19 & 0.070 & No \\
A21 & 0.146 & No & P20 & 0.070 & No \\
A22 & 0.514 & Yes & P21 & 0.284 & Yes \\
A23 & 0.070 & No & P22 & 0.284 & Yes \\
A24 & 0.070 & No & P23 & 0.202 & No \\
& 0.486 & Yes & P24 & 0.306 & Yes
\end{tabular}




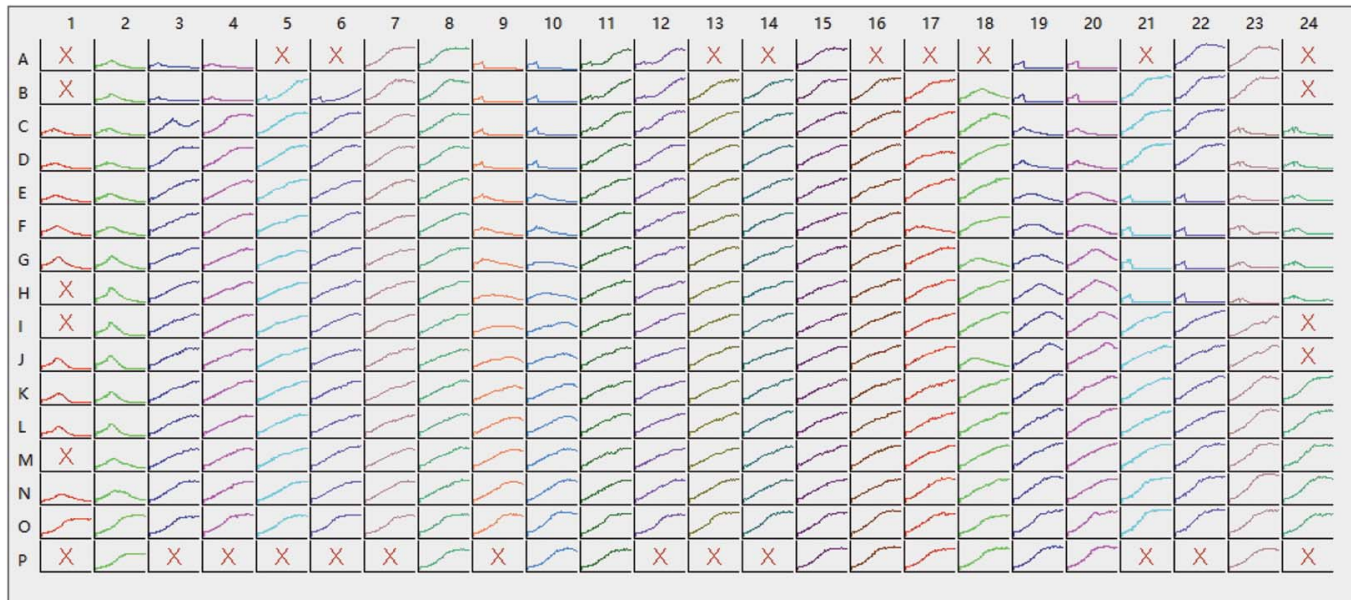

Fig. 5 Screening results of actual experiments.

$$
n=\left[\frac{\bar{T} T^{m}}{\bar{T}+T^{m}}\right]
$$

where $\bar{T}$ and $T^{m}$ are the sample size of mean TCRC and TCRC of the edge well. In this work, the sample size was fixed at 72, so $n=$ 32. $\alpha$ was the level of significance, in general $\alpha=0.05$ (95\% confidence). The flowchart of the algorithm is illustrated in Table 1.

\section{Case studies}

Data from four experiments were collected from February 2016 (Fig. 3). The box plots of results are shown in Fig. 4. The average of the inner curves is shown as a bold purple curve. As shown in two figures above, in many cases it is difficult to identify the edge effect through artificial selection. In this section, experiment 1 (2016-02-03) was used as an example to show the distinguishing procedure. Using the proposed method, the significance level was set as $\alpha=0.05$ and the critical region was $D_{n, 1-\alpha}=D_{36,1-0.05}=0.22119$. The calculation of the empirical distribution function is shown in Table 2. From eqn (8), the result is shown in Table 3 .

As shown in Table 2, the values of the statistical magnitude $D^{m}$ of the four edge-well TCRCs were calculated, which were greater than the critical region $D_{36,1-0.05}=0.22119$ (Table 3). According to the Smirnov test, hypothesis $H_{0}$ was rejected. Therefore, the edge effects occurred in four edge-well TCRCs of experiment 1 and those TCRCs should be removed before the cytotoxicity assay.

The results of three other experiments are shown in Table 4. It can be seen there are many edge-effect TCRCs, which are consistent with the actual situation (Fig. 3 and 4). Therefore, the proposed method could effectively detect the edge effect.

To compare the effects of different levels of significance of the experiment, another significant level was applied where $\alpha=$ 0.1 . The corresponding critical region was $D_{36,1-0.1}=0.19910$. The results are shown in Table 5.

The P22 of experiment 2 (2016-02-09) was distinguished as an edge effect with $\alpha=0.1$. However, from Fig. 3, the TCRC of
P22 fluctuated up and down with a mean curve in a small range, which is common when taking real cell growth into account. Therefore, in this work, it was too harsh to set $\alpha=0.1$, accordingly, in this work $\alpha=0.05$ was recommended.

Furthermore, the wells with chemicals were also tested by using the presented Simonov test. As mentioned before, each concentration of each chemical was repeated four times, and only two inner wells were set as references. The results were shown in Table 6. Based on the proposed algorithm, the wells with edge-effect were screened out (" $\times$ " in Fig. 5) automatically, which can reduce the technician's workload and remove the careless operation.

\section{Conclusion}

The primary goal of this work was to develop an effective method to detect the edge effect, which can help technicians rapidly screen valid TCRCs. A standard Smirnov test was used to determine this. Although the statistical methods employed are not new, it is the first time they were applied to determine the edge effect in RTCA systems. The method effectively determined the edge effect, and reduced the risk of error by manual screening.

Although the results demonstrated the effectiveness of the proposed method, future research should include the following:

- The choice of significant levels should be discussed based on the specific data, since the performance of this method is affected by the range interval;

- Other edge wells should be tested in addition to the negative control;

- Except for the TCRC of edge wells, the abnormal TCRC of inner wells with certain screening strategies should be discussed.

\section{Acknowledgements}

The work was supported by the National Nature Science Foundation of China [grant number 61273142], the Priority Academic Program Development of Jiangsu Higher Education Institutions (PAPD) and Foundation for Six Talents by Jiangsu 
Province. We also thank Xiao Xu, Xiaobo Wang and Can Jin from ACEA Biosciences Inc., David Kinniburgh and Dorothy Yu Huang from Alberta Centre for Toxicology, Weiping Zhang from Alberta Health, Biao Huang from University of Alberta (Canada) for scientific advice and technical support.

\section{References}

1 Organization W H., WHO human health risk assessment toolkit, chemical hazards, World Health Organization, 2010.

2 D. McGregor, A. Boobis, M. Binaglia, et al., Guidance for the classification of carcinogens under the Globally Harmonised System of Classification and Labelling of Chemicals (GHS), Crit. Rev. Toxicol., 2010, 40(3), 245-285.

3 F. Zucco, I. D. Angelis, E. Testai, et al., Toxicology investigations with cell culture systems: 20 years after, Toxicol. in Vitro, 2004, 18(2), 153-163.

4 T. Voskoglounomikos, J. L. Pater and L. Seymour, Clinical predictive value of the in vitro cell line, human xenograft, and mouse allograft preclinical cancer models, Clin. Cancer Res., 2003, 9(11), 4227-4239.

5 W. J. Pichler, Predictive drug allergy testing: an alternative viewpoint, Toxicology, 2001, 158(1-2), 31-41.

6 K. A. Houck and R. J. Kavlock, Understanding mechanisms of toxicity: insights from drug discovery research, Toxicol. Appl. Pharmacol., 2008, 227(2), 163-178.

7 Roche. xCELLigence system application note No. 16, RTCA HT instrument high throughput GPCR screening, Roche Diagnostics GmbH, Mannheim, Germany, 2011.

8 F. A. Atienzar, K. Tilmant, H. H. Gerets, et al., The use of realtime cell analyzer technology in drug discovery: defining optimal cell culture conditions and assay reproducibility with different adherent cellular models, J. Biomol. Screening, 2011, 16(6), 575-587.

9 T. H. Pan, B. Huang, J. Z. Xing, et al., Recognition of chemical compounds in contaminated water using time-dependent multiple dose cellular responses, Anal. Chim. Acta, 2012, 724(8), 30-39.
10 T. Zheng, X. Kuang, J. Wang, et al., Real-time cell analysis a new method for dynamic, quantitative measurement of infectious viruses and antiserum neutralizing activity, $J$. Virol. Methods, 2013, 193(2), 364-370.

11 J. Z. Xing, S. Gabos, B. Huang, et al., High-throughput quantitative analysis with cell growth kinetic curves for low copy number mutant cells, Anal. Bioanal. Chem., 2012, 404(6-7), 2033-2041.

12 L. Otero-González, R. Sierra-Alvarez, S. Boitano, et al., Application and validation of an impedance-based real time cell analyzer to measure the toxicity of nanoparticles impacting human bronchial epithelial cells, Environ. Sci. Technol., 2012, 46(18), 10271-10278.

13 Eli Lilly and Company and the National Institute of Health Chemical Genomics Center, Guidance for Assay Development \& HTS, March 2007, Version 5, 2005.

14 B. K. Lundholt, K. M. Scudder and L. Pagliaro, A Simple Technique for Reducing Edge Effect in Cell-Based Assays, J. Biomol. Screening, 2003, 8(5), 566-570.

15 B. J. Foley, A. M. Drozd, M. T. Bollard, et al., Maintaining Microclimates during Nanoliter Chemical Dispensations Using Custom-Designed Source Plate Lids, JALA, 2015, 21(1), 115-124.

16 N. Malo, J. A. Hanley, S. Cerquozzi, et al., Statistical practice in high-throughput screening data analysis, Nat. Biotechnol., 2006, 24(2), 167-175.

17 J. Chen, T. H. Pan, B. D. Devendran, et al., Analysis of inter-/ intra-E-plate repeatability in the real-time cell analyzer, Anal. Biochem., 2015, 477, 98-104.

18 T. H. Pan, H. R. Li, K. Swanand, et al., High-throughput screening assay for the environmental water samples using cellular response profiles, Ecotoxicol. Environ. Saf., 2015, 114, 134-142.

19 L. Mora-López and J. Mora, An adaptive algorithm for clustering cumulative probability distribution functions using the Kolmogorov-Smirnov two-sample test, Int. J. Expert Sys. Appl., 2015, 42(8), 4016-4021. 